\title{
CT-Scan Evaluation for the Detection of Benign Parapharyngeal Mass
}

\author{
Md. Abdul Alim¹, Rokshana Ahmed², Zerin Haque ${ }^{3}$ Rafiqul Islam4, Rownak Jahan Jowarder 5 \\ Meher Angez Rahman ${ }^{6}$, Nazmun Nahar ${ }^{7}$ \\ ${ }^{1}$ Assistant Professor, Department of Neuro-radiology and Imaging, National Institute of Neurosciences and Hospital, Dhaka, \\ Bangladesh; ${ }^{2}$ Assistant Professor, Department of Neuro-radiology and Imaging, National Institute of Neurosciences and \\ Hospital, Dhaka, Bangladesh; ${ }^{3}$ Junior Consultant, Department of Neuro-radiology and Imaging, National Institute of \\ Neurosciences and Hospital, Dhaka, Bangladesh; ${ }^{4}$ Associate Professor, Department of Pharmacology, National Institute \\ of Neurosciences \& Hospital, Dhaka, Bangladesh; ${ }^{5}$ Post-Graduate Student [M. Phil, Thesis Part], Department of \\ Pharmacology, Mymensingh Medical College, Mymensingh, Bangladesh; ${ }^{6}$ Radiologist, Suri Seri Begawan \\ Hospital, Kuala Belait, Brunei; ${ }^{7}$ Associate Professor \& Head, Department of Neuro-radiology and Imaging, \\ National Institute of Neurosciences and Hospital, Dhaka, Bangladesh
}

[Received on: 22 April 2021; Accepted on: 12 May 2021; Published: 1 July 2021]

\begin{abstract}
Background: Detection of benign parapharyngeal mass is very crucial for the management of the patients. Objective: The purpose of the present study was to evaluate the CT scan for the detection of benign parapharyngeal mass. Methodology: This cross-sectional study was carried out in the Department of Radiology and Imaging at Bangabandhu Sheikh Mujib Medical University, Dhaka, Bangladesh from July 2009 to April 2011 for a period of around two years. All the clinically suspected cases of parapharyngeal space mass lesions attended in the Otolaryngology and Radiology \& Imaging department of Bangabandhu Sheikh Mujib Medical University (BSMMU), Dhaka were enrolled in this study. The patient in this study was evaluated according to the management plan for surgery followed in Otolaryngology department. The histopathology was performed by the histopathologist of the Department of Pathology at BSMMU, Dhaka, Bangladesh. All patients were undergone CT examination of neck and the comparison of the findings was done with the histopathology results. Result: A total number of 50 patients were recruited for this study after fulfilling the inclusion and exclusion criteria. Mean $( \pm \mathrm{SD})$ age of the patients was $37.76( \pm 14.82)$. Sensitivity of CT to diagnose benign parapharyngeal mass was $92.1 \%$, specificity $83.3 \%$, positive predictive value $94.6 \%$, negative predictive value $76.9 \%$ and accuracy $90.0 \%$. The area under the curve value was calculated and was found $0.142(95 \%$ CI 0.000 to 0.286$)(\mathrm{p}=0.000)$. Conclusion: In conclusion the sensitivity and specificity of CT scan for the detection of benign parapharyngeal mass lesion is very high. [Journal of National Institute of Neurosciences Bangladesh, July 2021;7(2):118-121]
\end{abstract}

Keywords: Evaluation; CT-Scan; Benign Parapharyngeal Mass

Correspondence: Dr. Md. Abdul Alim, Assistant Professor, Department of Radiology and Imaging, National Institute of Neurosciences and Hospital, Sher-E-Bangla Nagar, Agargaon, Dhaka-1207, Bangladesh; Email: aurinryne@gmail.com; Cell no.: $+8801816862167$

Conflict of interest: There is no conflict of interest relevant to this paper to disclose.

Funding agency: This research project was not funded by any group or any institution.

Contribution to authors: Alim MA, Ahmed R, Haque Z, Nahar N were involved in protocol preparation, data collection and literature search and manuscript writing. Islam R, Jowarder RJ, Rahman MA were involved in preparation and revision of this manuscript.

How to cite this article: Alim MA, Ahmed R, Haque Z, Islam R, Jowarder RJ, Rahman MA, Nahar N. CT-Scan Evaluation for the Detection of Benign Parapharyngeal Mass. J Natl Inst Neurosci Bangladesh, 2021;7(2): 118-121

Copyright: (C2021. Alim et al. Published by Journal of National Institute of Neurosciences Bangladesh. This article is published under the Creative Commons CC BY-NC License (https://creativecommons.org/licenses/by-nc/4.0/). This license permits use, distribution and reproduction in any medium, provided the original work is properly cited, and is not used for commercial purposes.

\section{Introduction}

Tumors originating in parapharyngeal space are rare, accounting for $0.5 \%$ of head \& neck neoplasia ${ }^{1}$ and present a formidable challenge to the surgeon with regard to both their pre-operative diagnosis and the specific technique of their surgical excision. There has been considerable improvement in imaging technique in the recent years for accurate pre-operative evaluation of these tumors. This process has helped in knowing the extent of tumor as well as establishing the preoperative 
histological diagnosis and tumor behavior in early stage ${ }^{2}$. Expansion of the tumor occurs in the plane of least resistance namely, medially towards the tonsil and lateral pharyngeal wall, laterally between the tail of parotid and sub mandibular gland and posteriorly into retro mandibular area ${ }^{3}$. The diagnosis should be suspected when a mass is encountered that displaces the tonsil/lateral pharyngeal wall towards mid line or when a mass is encountered in the neck near the angle of mandible. Tumors which are palpable in the neck may pass through the stylomandibular tunnel and are of dumb-bell shape usually benign pleomorphic adenoma ${ }^{4}$. Other manifestations depends on site of origin and the effect upon surrounding structures like otological hoarseness of voice, deviation of tongue, Homer's syndrome. Trismus is a common feature which implies either irritation of the pterygoid muscle or perhaps mechanical obstruction to motion of mandible ${ }^{5}$.

CT-scan with intravenous contrast and enhancement is the most useful radiographic modality currently employed $^{6}$. It determines the location extent, vascularity of tumor with differentiation of parotid and extra parotid region by presence or absence of lucent line which represents the fibro-fatty matrix of the parapharyngeal space ${ }^{7}$. This present study was undertaken to evaluate the CT scan for the detection of benign parapharyngeal mass.

\section{Methodology}

This was a cross sectional type of study. This study was carried out in the Department of Radiology and Imaging, Bangabandhu Sheikh Mujib Medical University, Dhaka, Bangladesh. This study was carried out from July 2009 to April 2011 for a period of two years. All the clinically suspected cases of parapharyngeal space mass lesions attended in the Otolaryngology and Radiology \& Imaging department of Bangabandhu Sheikh Mujib Medical University (BSMMU), Dhaka were enrolled in this study. Samples were selected purposively. Patient having clinical suspicion of parapharyngeal space mass lesions who undergone CT scan at any age group irrespective of their sex were included as study population. Patients who were not fit for surgery or patients whose histopathology reports were not available due to lost to follow up were excluded this study. The patient in this study was evaluated according to the management plan for surgery followed in Otolaryngology department, which included history, clinical examination. The histopathology was performed by the histopathologist of the Department of Pathology at BSMMU, Dhaka,
Bangladesh. All patients were undergone CT examination of neck in the Department of Radiology and Imaging, BSMMU, Dhaka and the diagnosis of benign mass was done based on standard criteria. CT scan was performed with a third generation CT machine, Hitachi W2000. (3-5) mm thick contiguous slice was taken. These scans were obtained using 120 $\mathrm{kV}, 150 \mathrm{~mA}$ and $1 \mathrm{sec}$ scanning time for 1 slice. From skull base to root of the neck was scanned. Both pre and post contrast scans were performed. $50 \mathrm{ml}$ of non-ionic water soluble iodinated contrast media of 370 strength was administered. Immediately after completion of bolus injection 3-5 mm contiguous slice was obtained. After informing all the necessary information regarding the research study, data were collected in a pre-designed structured data collection sheets. Statistical analysis of the data was obtained by using windows based computer software device with Statistical Packages for Social Sciences (SPSS-13). For the validity of study outcome, specificity (defined as the proportion of true negatives correctly identified by the test indicating how often a tumor-like lesion is diagnosed correctly), sensitivity (defined as the proportion of true positives correctly identified by the test indicating how often a non-tumor-like lesion is diagnosed correctly) and the accuracy (defined as the proportion of true negatives and true positives correctly identified by the test; indicating the agreement between the preoperative diagnosis and the histological outcome) were calculated.

\section{Results}

A total number of 50 patients were recruited for this study after fulfilling the inclusion and exclusion criteria. Out of all patients maximum $(54.0 \%)$ were belonged to 21 to 40 years age group followed by 41 and above age group (38.0\%) and up to 20 years age group $(8.0 \%)$. Mean $( \pm \mathrm{SD})$ age of the patients was $37.76( \pm 14.82)$ (Table 1$)$.

Table 1: Distribution of the patients by age $(n=50)$

\begin{tabular}{lcc}
\hline Age Group & Frequency & Percent \\
\hline$\leq 20$ Years & 4 & 8.0 \\
21 to 40 Years & 27 & 54.0 \\
41 and above Years & 19 & 38.0 \\
Total & $\mathbf{5 0}$ & $\mathbf{1 0 0 . 0}$ \\
\hline
\end{tabular}

Mean \pm SD $($ Range $)=37.76 \pm 14.82(2.7-72.0)$

Out of all patients 35 cases were diagnosed as benign parapharyngeal mass by $\mathrm{CT}$ and confirmed by histopathological evaluation. They were true positive. 2 
cases were diagnosed as benign parapharyngeal mass by CT but not confirmed by histopathological findings. They were false positive. Of 13 cases of other than benign parapharyngeal mass, which were diagnosed by CT, and 3 were confirmed as benign mass and 10 other than masses by histopathology. Therefore, false positive cases and true negative cases were 2 and 10 respectively (Table 2 ).

Table 2: Relationship between CT scan and Histopathological diagnosis of benign parapharyngeal mass $(n=50)$

\begin{tabular}{lccc}
\hline CT scan & \multicolumn{2}{c}{ Histopathological diagnosis } & Total \\
\cline { 2 - 3 } & Benign & Others & \\
\hline Benign & 35 & 2 & 37 \\
Others & 3 & 10 & 13 \\
Total & $\mathbf{3 8}$ & $\mathbf{1 2}$ & $\mathbf{5 0}$ \\
\hline
\end{tabular}

Sensitivity of CT to diagnose benign parapharyngeal mass was $92.1 \%$ (95\% CI $78.62 \%$ to $98.34 \%)$, specificity $83.3 \% \quad(95 \%$ CI $51.59 \%$ to $97.91 \%)$, positive predictive value $94.6 \%$ (95\% CI $83.11 \%$ to $98.42 \%)$, negative predictive value $76.9 \%$ (95\% CI $52.22 \%$ to $91.04 \%)$ and accuracy $90.0 \%$ (95\% CI $78.19 \%$ to $96.67 \%$ ) (Table 3 ).

Table 3: Validity of CT scan in the Diagnosis of Benign Parapharyngeal Mass

\begin{tabular}{lcc}
\hline Validity & Values & 95\% CI \\
\hline Sensitivity & 92.1 & $78.62 \%$ to $98.34 \%$ \\
Specificity & 83.3 & $51.59 \%$ to $97.91 \%$ \\
PPV & 94.6 & $83.11 \%$ to $98.42 \%$ \\
NPV & 76.9 & $52.22 \%$ to $91.04 \%$ \\
Accuracy & 90.0 & $78.19 \%$ to $96.67 \%$ \\
\hline
\end{tabular}

PPV = Positive Predictive Value; NPV = Negative Predictive Value

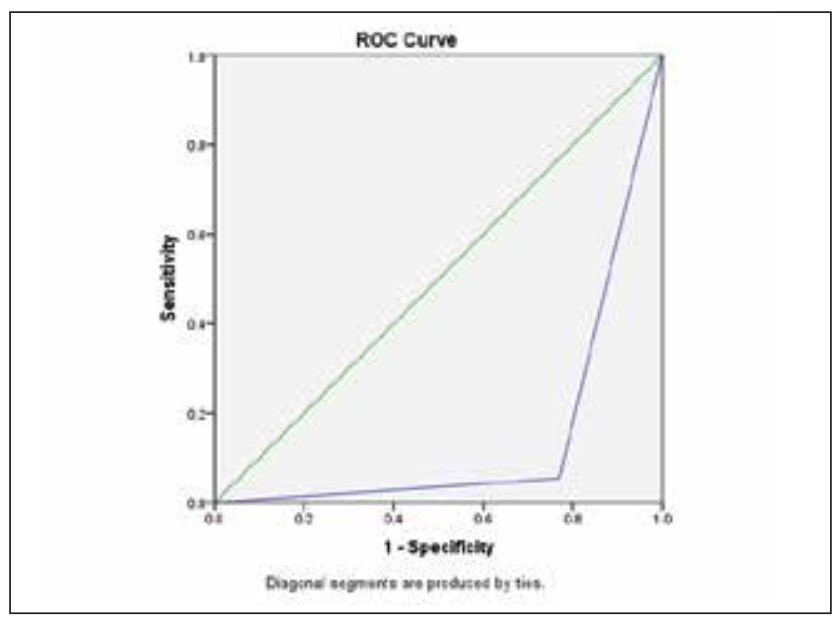

Figure I: Showing the ROC Curve of CT scan for detection of Benign Parapharyngeal Mass
The area under the curve value was calculated and was found 0.142 (95\% CI 0.000 to 0.286$)(p=0.000)$ (Table 4).

Table 4: Area under the curve Value of CT scan for detection of Benign Parapharyngeal Mass

\begin{tabular}{lccc}
\hline AUC Value & P value & \multicolumn{2}{c}{ Asymptotic 95\% CI } \\
\cline { 3 - 4 } & & Lower & Upper \\
\hline 0.142 & 0.000 & 0.000 & 0.286 \\
\hline
\end{tabular}

\section{Discussion}

With advent of high resolution CT-scan, operative facilities in form of electro-cautery, hypotensive anaesthesia, para pharyngeal tumors are now amenable to proper management ${ }^{8}$. The presentation of para-pharyngeal space tumor is usually benign and produces symptoms by exerting pressure on neighboring structures ${ }^{9}$. Out of all patients 35 cases were diagnosed as benign parapharyngeal mass by CT-scan and confirmed by histopathological evaluation. They were true positive. 2 cases were diagnosed as benign parapharyngeal mass by $\mathrm{CT}$ but not confirmed by histopathological findings. They were false positive. Of 13 cases of other than benign parapharyngeal mass, which were diagnosed by CT-scan and 3 cases were confirmed as benign mass and 10 other than masses by histopathology. Therefore, false positive cases and true negative cases were 2 cases and 10 cases respectively.

Izzo et $\mathrm{al}^{10}$ have reported that among the parapharyngeal space (PPS) tumors, 70.0 to $80.0 \%$ are benign, and 20.0 to $30.0 \%$ are malignant. Most parapharyngeal space (PPS) tumors are of salivary or neurogenic origin, although metastatic lesions; lymphoreticular lesions; and a variety of uncommon, miscellaneous lesions may arise in this location.

Ahmad et $\mathrm{al}^{11}$ determined the role of clinical features, fine needle aspiration cytology (FNAC) and computed tomography (CT) scan in diagnosing parapharyngeal space (PPS) tumors and treatment options. They reviewed the medical record of patients for their age, gender, clinical features, investigations (FNAC and CT scan) and treatment. The mean age of patients presenting with PPS tumours was 33.6 years. The most common clinical features were neck mass (93\%) and bulge in lateral pharyngeal wall $(80 \%)$. The CT scan showed exact location and extent of tumour in 11 out of 15 cases. The sensitivity and specificity of FNAC was $70 \%$ and $85 \%$ respectively.

Surgical and CT examinations have been performed on 104 patients and each of whom presented with a parapharyngeal space mass, has resulted in the 
development of an updated $\mathrm{CT}$ protocol designed to provide a preoperative diagnosis ${ }^{12}$. When dynamic scanning is used, diagnostic angiography or digital venous imaging (DVI) can be avoided in almost all of these cases. A specific preoperative diagnosis can be made in $88.0 \%$ of the patients and a limited differential diagnosis, which includes the final diagnosis, can be made in virtually all cases ${ }^{13}$.

Sensitivity of CT to diagnose benign parapharyngeal mass was $92.1 \%(95 \%$ CI $78.62 \%$ to $98.34 \%)$, specificity $83.3 \%$ (95\% CI $51.59 \%$ to $97.91 \%$ ), positive predictive value $94.6 \%$ (95\% CI $83.11 \%$ to $98.42 \%$ ), negative predictive value $76.9 \%$ (95\% CI $52.22 \%$ to $91.04 \%)$ and accuracy $90.0 \%$ (95\% CI $78.19 \%$ to $96.67 \%)$. Similar result has been published different studies. In a study ${ }^{14,51}$ patients underwent surgery for parapharyngeal space tumors. Eighty percent of the parapharyngeal space neoplasms were benign; $20 \%$ were malignant. Fifty-seven percent $(31 / 54)$ were of neurogenic origin, $30 \%(16 / 54)$ were of salivary origin, and $13 \%(7 / 54)$ were of miscellaneous origin. The use of computed tomography scan and magnetic resonance imaging, and selective use of angiography, allowed us to ascertain the location, size, vascularity, and relation of parapharyngeal space tumors to surrounding anatomical structures. Imaging techniques established the site of origin of these tumors with $96 \%$ accuracy ${ }^{14}$.

Prior to the CT era, very limited radiologic information could be gleaned from plain films and multidirectional tomogram $\mathrm{s}^{15}$. The degree of lesion vascularity could be identified on a conventional angiogram; however, only a lesion such as a paraganglioma, with its characteristic hypervascular appearance, or an aneurysm could be confidently diagnosed ${ }^{12}$. A lesion could also be identified as being hypovascular, but a salivary gland tumor could not be differentiated from a schwannoma or other such pathology.

\section{Conclusion}

In conclusion the sensitivity of CT scan is very high for the detection capacity of benign parapharyngeal mass. Furthermore the specificity of CT scan for the detection of benign parapharyngeal mass lesion is very high. Therefore this CT scan is very good diagnostic utility for the detection of benign tumor mass in the parapharyngeal space. Further multicenter study should be conducted to get the more accurate result.

\section{References}

1. Vu TH, Kwon M, Ahmed S, Gule-Monroe M, Chen MM, Sun J, Fornage BD, Debnam JM, Edeiken-Monroe B. Diagnostic Accuracy and Scope of Intraoperative Transoral Ultrasound and Transoral Ultrasound-Guided Fine-Needle Aspiration of Retropharyngeal Masses. American Journal of Neuroradiology. 2019;40(11):1960-4 2. Khafif A, Segev Y, Kaplan DM, Gil ZI, Fliss DM. Surgical management of parapharyngeal space tumors: a 10-year review. Otolaryngology — Head and Neck Surgery. 2005;132(3):401-6.

3. Stambuk HE, Patel SG. Imaging of the parapharyngeal space. Otolaryngologic Clinics of North America. 2008;41(1):77-101.

4. Eisele DW, Richmon JD. Contemporary evaluation and management of parapharyngeal space neoplasms. The Journal of Laryngology \& Otology. 2013;127(6):550-5.

5. Gupta A, Chazen JL, Phillips CD. Imaging evaluation of the parapharyngeal space. Otolaryngologic Clinics of North America. 2012;45(6):1223-32.

6. Varoquaux A, Fakhry N, Gabriel S, Garcia S, Ferretti A, Chondrogiannis S, Rubello D, Taïeb D. Retrostyloid parapharyngeal space tumors: a clinician and imaging perspective. European Journal of Radiology. 2013;82(5):773-82.

7. Liyanage SH, Spencer SP, Hogarth KM, Makdissi J. Imaging of salivary glands. Imaging. 2007;19(1):14-27.

8. Handa U, Bal A, Mohan H, Dass A. Parapharyngeal paraganglioma: diagnosis on fine-needle aspiration. American Journal of Otolaryngology. 2005;26(5):360-1.

9. Liu Y, Li J, Tan YR, Xiong P, Zhong LP. Accuracy of diagnosis of salivary gland tumors with the use of ultrasonography, computed tomography, and magnetic resonance imaging: a meta-analysis. Oral Surgery, Oral Medicine, Oral Pathology and Oral Radiology. 2015;119(2):238-45.

10. Izzo L, Casullo A, Caputo M, Costi U, Guerrisi A, Stasolla A, Basso L, Marini M, De Toma G. Space occupying lesions of parotid gland. Comparative diagnostic imaging and pathological analysis of echo color/power Doppler and of magnetic resonance imaging. Acta Otorhinolaryngologica Italica. 2006;26(3):147

11. Ahmad F, Uddin W, Khan Y, Khawar A, Bangush W, Aslam J. Management of Parapharyngeal space tumors. J Coll Physicians Surg Pak 2006;16(1):7-10

12. Koyuncu M, Sesen T, Akan H, Ismailoglu AA, Tanyeri Y, Tekat A, Unal R. Comparison of computed tomography and magnetic resonance imaging in the diagnosis of parotid tumors. Otolaryngol Head Neck Surg 2003;129:726-32

13. Rudack C, Jörg S, Kloska S, Stoll W, Thiede O. Neither MRI, CT nor US is superior to diagnose tumors in the salivary glands-an extended case study. Head \& face medicine. 2007 Dec;3(1):19

14. Jin GQ, Su DK, Xie D, Zhao W, Liu LD, Zhu XN. Distinguishing benign from malignant parotid gland tumours: low-dose multi-phasic CT protocol with 5-minute delay. European radiology. 2011 Aug $1 ; 21(8): 1692-8$

15. Park SB, Choi JY, Lee EJ, Yoo J, Cheon M, Cho SK, Choe YS, Lee KH, Kim BT. Diagnostic criteria on 18 F-FDG PET/CT for differentiating benign from malignant focal hypermetabolic lesions of parotid gland. Nuclear medicine and molecular imaging. 2012 Jun 1;46(2):95-101 